\title{
Uniquely Solvable Problems for Abstract Legendre Equation
}

\author{
A. V. Glushak ${ }^{1 *}$ \\ ${ }^{1}$ Belgorod State National Research University \\ ul. Pobedy 85, Belgorod, 308015 Russia \\ Received April 15, 2017
}

\begin{abstract}
For loaded abstract Legendre equation we find sufficient conditions of solvability of the Cauchy problem and the boundary control problem. We also consider nonlocal problem that contains fractional integral of a function with respect to another function.
\end{abstract}

DOI: $10.3103 / \mathrm{S} 1066369 \mathrm{X} 18070010$

Keywords: abstract Legendre equation, Cauchy problem, unique solvability, nonlocal condition, fractional integral of a function with respect another function.

The research of some physical processes relies on solving of equations with the Laplace operator, which (by means of separation of variables in curvilinear coordinate systems) leads to differential equations containing singularity. If there is a symmetry of some sort, then such equations turn into the Euler-Poisson-Darboux (EPD) equation and the Legendre equation.

Initial problems for the classic and abstract EPD equation were studied in a series of papers and the results are presented in [1] (Chap. 1). The further results in this direction were obtained in author's papers $[2,3]$.

In this paper we will consider some additional conditions and solvability of corresponding problems for one more abstract singular equation, namely, the Legendre equation.

Let $A$ be a closed operator in Banach space $E$ with domain $D(A)$ which is dense in $E$. When $k>0$ let us consider Legendre equation

$$
L_{k} u(t) \equiv u^{\prime \prime}(t)+k \operatorname{coth} t u^{\prime}(t)+(k / 2)^{2} u(t)=A u(t), \quad t>0 .
$$

Differential operator $L_{k}$ in the left-hand side of Eq. (1) occurs when solving the Laplace equation in elongated ellipsoid of revolution coordinates ([4], P. 138). If $A$ is a scalar multiplication operator, then the spherical functions considered in [5] (P. 53) satisfies Eq. (1) where $k=2$. We also note papers [6-11] that study partial differential equations with singular operator of the considered type.

As follows from result in [12], the correct formulation of the initial conditions for abstract Legendre equation (1) consists in setting the initial conditions

$$
u(0)=u_{0}, \quad u^{\prime}(0)=0,
$$

in the point $t=0$. Herewith, if $k \geq 1$, then the initial condition $u^{\prime}(0)=0$ is removed, which is a characteristic for a number of equations with a singularity in coefficients when $t=0$.

When $k=0$, problem (1), (2) is uniformly correct if and only if operator $A$ is a generator of cosineoperator-function (COF) $C(t)$ and we will write down this fact as $A \in G_{0}$ (see terminology in [13, 14]).

In [12] there are conditions on operator $A$ which provide correct solvability of problem (1), (2). We will denote by $G_{k}$ the set of operators $A$ with which problem (1), (2) is uniformly correct, the resolving operator of this problem we will denote by $P_{k}(t)$ and call it the operator Legendre function (OLF).

OLF $P_{k}(t)$ which was introduced in [12] was used by the author in [15] when establishing the criterion of stabilization of the Cauchy problem for abstract differential first-order equation solution. It can also be used for solution of the weight Cauchy problem for Legendre equation. If $0<k<1$, then there is

\footnotetext{
*E-mail: aleglu@mail.ru.
} 
a correct statement of the initial conditions which is more general than in (2). Let us consider initial conditions

$$
u(0)=u_{0}, \lim _{t \rightarrow 0}\left(\frac{\sinh t}{t}\right)^{k} u^{\prime}(t)=u_{1}
$$

When $u_{0}, u_{1} \in D(A)$ and $A \in G_{k} \subset G_{2-k}$, the unique solution to the Cauchy problem (1), (3) has the form [12]

$$
u(t)=P_{k}(t) u_{0}+\frac{1}{1-k}\left(\frac{\sinh t}{t}\right)^{1-k} P_{2-k}(t) u_{1} .
$$

Note that if $A \in G_{k}$ and $k \geq 1$, then problem (1),(3) is not correct. In what follows we study another statements of additional conditions, which allow us to establish the unique solvability of corresponding problems for the loaded Legendre equation.

1. The Cauchy problem for weakly loaded Legendre equation. Let us consider equation

$$
u^{\prime \prime}(t)+k \operatorname{coth} t\left(u^{\prime}(t)-\frac{\cosh ^{2-k}(t / 2)}{\cosh t} u^{\prime}(0)\right)+\frac{k^{2}}{4} u(t)=A u(t), \quad t>0,
$$

which, unlike Eq. (1), contains the value of the derivative of an unknown function in point $t=0$. We will call Eq. (4) the weakly loaded Legendre equation (see terminology in the introduction to monographs $[16,17])$. The growing interest in research of loaded differential equations is caused by an expanding number of their applications and the fact that loaded equations form a distinct class of functionaldifferential equations with specific problems. The review of papers on the loaded differential equations can be also found in $[16,17]$.

It is significant that in Eq. (4) there is a load given when $t=0$ and this fact changes the statement of the initial problem. Unlike weight problem (1), (3), when $k>0$ we will establish the correctness of Cauchy problem

$$
u(0)=u_{0}, u^{\prime}(0)=u_{1}
$$

for weakly loaded Eq. (4) and specify the resolving operator explicitly.

Further we will assume that $g(t)=\cosh t$. Let us consider the fractional integral of function $f(t)$ with respect to function $g(t)=\cosh t([18]$, P. 248)

$$
I_{g}^{\alpha} f(t)=\frac{1}{\Gamma(\alpha)} \int_{0}^{t}(\cosh t-\cosh s)^{\alpha-1} \sinh s f(s) d s .
$$

We also assume that $\mu_{k}=\frac{2^{k / 2} \Gamma(k / 2+1 / 2)}{\sqrt{\pi} \Gamma(k / 2)}$ and use notation $P_{k}^{\prime}(t) u_{0}=\left(P_{k}(t) u_{0}\right)^{\prime}$ for brevity.

Theorem 1 ([12]). Let operator $A$ be a generator of $C O F C(t), u_{0} \in D(A)$. Then problem (1), (2) is uniformly correct, i.e., $A \in G_{k}$, and the corresponding $O L F$ can be represented as

$$
P_{k}(t) u_{0}=\mu_{k} \sinh ^{1-k} t \int_{0}^{t}(\cosh t-\cosh s)^{k / 2-1} C(s) u_{0} d s=\mu_{k} \Gamma(k / 2) \sinh ^{1-k} t I_{g}^{k / 2}\left[\frac{C(t)}{\sinh t}\right] u_{0},
$$

and

$$
P_{k}^{\prime}(t) u_{0}=\frac{\sinh t}{k+1} P_{k+2}(t)\left(A-\frac{k^{2}}{4} I\right) u_{0}
$$

According to Theorem 1 , if $u_{1}=0$, then function $u(t)=P_{k}(t) u_{0}$ from (6) is the unique solution to the Cauchy problem (4), (5). 
In particular case when operator $A=(\delta+1 / 2)^{2}, \delta \in \mathbb{R}$ is a multiplication by number operator, OLF $P_{k}(t)$ can be expressed through attached Legendre function of the first kind $P_{\delta}^{\beta}(\cdot)$ ([19], P. 661)

$$
P_{k}(t)=\Gamma(1-\beta)\left(\frac{1}{2} \sinh t\right)^{\beta} P_{\delta}^{\beta}(\cosh t), \beta=\frac{1-k}{2} .
$$

Hereinafter we will need the following equations from [12], which was used in the proof of Theorem:

$$
\begin{gathered}
L_{k}\left(\sinh ^{1-k} t u(t)\right)=\sinh ^{1-k} t L_{2-k} u(t), \\
\left(\frac{1}{\sinh t} \frac{d}{d t}\right)^{-\alpha} u(t)=\frac{1}{\Gamma(\alpha)} \int_{0}^{t}(\cosh t-\cosh s)^{\alpha-1} \sinh s u(s) d s=I_{g}^{\alpha} u(t), \alpha>0 ;
\end{gathered}
$$

this is a definition of negative fractional exponent of the operator of weight differentiation, which with respect to connection with fractional integral with respect to function $g(t)=\cosh t$ can be extended ([18], P. 248) on all $\alpha \in \mathbb{R}$; if $u(0)=0$, then

$$
L_{k+2 \alpha}\left(\frac{1}{\sinh t} \frac{d}{d t}\right)^{\alpha} u(t)=\left(\frac{1}{\sinh t} \frac{d}{d t}\right)^{\alpha} L_{k} u(t),
$$

note that when $\alpha \in \mathbb{N}$ condition $u(0)=0$ is not applied.

Note that Eq. (10) means that we can apply to considered in this paper problems the method of transformation operator which is used in [1] (Chap. 2) and is a main method in papers [7, 11].

From (8)-(10) we can obtain

$$
\begin{gathered}
L_{k}\left(\sinh ^{1-k} t\left(\frac{1}{\sinh t} \frac{d}{d t}\right)^{-k / 2} \frac{u(t)}{\sinh t}\right)=\sinh ^{1-k} t L_{2-k}\left(\left(\frac{1}{\sinh t} \frac{d}{d t}\right)^{-k / 2} \frac{1}{\sinh t} \frac{d}{d t} \int_{0}^{t} u(\tau) d \tau\right) \\
=\sinh ^{1-k} t L_{2-k}\left(\left(\frac{1}{\sinh t} \frac{d}{d t}\right)^{1-k / 2} \int_{0}^{t} u(\tau) d \tau\right)=\sinh ^{1-k} t\left(\frac{1}{\sinh t} \frac{d}{d t}\right)^{1-k / 2} \frac{d^{2}}{d t^{2}} \int_{0}^{t} u(\tau) d \tau \\
=\sinh ^{1-k} t\left(\frac{1}{\sinh t} \frac{d}{d t}\right)^{1-k / 2}\left(\int_{0}^{t} u^{\prime \prime}(\tau) d \tau+u^{\prime}(0)\right) \\
=\sinh ^{1-k} t\left(\frac{1}{\sinh t} \frac{d}{d t}\right)^{-k / 2} \frac{u^{\prime \prime}(t)}{\sinh t}+\sinh ^{1-k} t\left(\frac{1}{\sinh t} \frac{d}{d t}\right)^{1-k / 2} u^{\prime}(0) .
\end{gathered}
$$

Let us calculate the term $\left(\frac{1}{\sinh t} \frac{d}{d t}\right)^{1-k / 2} u^{\prime}(0)$ from (11). If $0<k<2$, then by (9) we will get

$$
\begin{aligned}
\left(\frac{1}{\sinh t} \frac{d}{d t}\right)^{1-k / 2} & u^{\prime}(0)=\frac{1}{\sinh t} \frac{d}{d t}\left(\frac{1}{\sinh t} \frac{d}{d t}\right)^{-k / 2} u^{\prime}(0) \\
& =\frac{1}{\sinh t} \frac{d}{d t}\left(\frac{1}{\Gamma(k / 2)} \int_{0}^{t}(\cosh t-\cosh s)^{k / 2-1} \sinh s u^{\prime}(0) d s\right) \\
& =\frac{1}{\Gamma(k / 2+1) \sinh t} \frac{d}{d t}\left((\cosh t-1)^{k / 2}\right) u^{\prime}(0)=\frac{1}{\Gamma(k / 2)}(\cosh t-1)^{k / 2-1} u^{\prime}(0) .
\end{aligned}
$$

If $k=2$, then $\left(\frac{1}{\sinh t} \frac{d}{d t}\right)^{1-k / 2} u^{\prime}(0)=u^{\prime}(0)$. Finally, if $k>2$, then by (9)

$$
\left(\frac{1}{\sinh t} \frac{d}{d t}\right)^{1-k / 2} u^{\prime}(0)=\frac{1}{\Gamma(k / 2)}(\cosh t-1)^{k / 2-1} u^{\prime}(0) .
$$

Thus, (11) can be rewritten as

$$
L_{k}\left(\sinh ^{1-k} t I_{g}^{k / 2}\left[\frac{u(t)}{\sinh t}\right]\right)=\sinh ^{1-k} t I_{g}^{k / 2}\left[\frac{u^{\prime \prime}(t)}{\sinh t}\right]+\frac{\sinh ^{1-k} t}{\Gamma(k / 2)}(\cosh t-1)^{k / 2-1} u^{\prime}(0)
$$


Note that Eq. (12) is written for function $u(t)=C(t) u_{0}, u^{\prime \prime}(t)=A C(t) u_{0}, u^{\prime}(0)=0$ and was used in the proof of Theorem 1 . As we will see further, this equation determines the multiplier in load $u^{\prime}(0)$ in Eq. (4).

Now let us consider the Cauchy problem (4), (5) in the case when $u_{0}=0$. Let $\nu_{k}=k 2^{k / 2-1}$. We introduce the sine-operator-function (SOF) $S(t)=\int_{0}^{t} C(s) d s$.

Theorem 2. If $u_{0}=0, u_{1} \in D(A)$ and operator $A$ is a generator of $C O F C(t)$, then function $u(t)=Q_{k}(t) u_{1}$, where

$$
Q_{k}(t) u_{1}=\nu_{k} \sinh ^{1-k} t \int_{0}^{t}(\cosh t-\cosh \tau)^{k / 2-1} S(\tau) u_{1} d \tau=\nu_{k} \Gamma(k / 2) \sinh ^{1-k} t I_{g}^{k / 2}\left[\frac{S(t)}{\sinh t}\right] u_{1}
$$

is a solution to problem (4), (5), and

$$
Q_{k}^{\prime}(t) u_{1}=\frac{\sinh t}{k+2} Q_{k+2}(t)\left(A-\frac{k^{2}}{4} I\right) u_{1}+\frac{u_{1}}{\cosh ^{k}(t / 2)} .
$$

Proof. Let us verify that function $Q_{k}(t) u_{1}$ satisfies Eq. (4). In order to do this let us substitute function $u(t)=S(t) u_{1}, u^{\prime \prime}(t)=A S(t) u_{1}, u^{\prime}(0)=u_{1}$ in (11). After some elementary transformations we will get

$$
\begin{aligned}
L_{k} Q_{k}(t) u_{1} & =L_{k}\left(\nu_{k} \Gamma(k / 2) \sinh ^{1-k} t I_{g}^{k / 2}\left[\frac{S(t)}{\sinh t}\right] u_{1}\right) \\
& =\nu_{k} \Gamma(k / 2)\left(\sinh ^{1-k} t I_{g}^{k / 2}\left[\frac{A S(t)}{\sinh t}\right] u_{1}+\frac{\sinh ^{1-k} t}{\Gamma(k / 2)}(\cosh t-1)^{k / 2-1} u_{1}\right) \\
= & A Q_{k}(t) u_{1}+\frac{k 2^{k}\left(2 \sinh ^{2}(t / 2)\right)^{k / 2-1}}{\sinh t(2 \sinh (t / 2) \cosh (t / 2))^{k-2}} u_{1}=A Q_{k}(t) u_{1}+k \operatorname{coth} t \frac{\cosh ^{2-k}(t / 2)}{\cosh t} u_{1}
\end{aligned}
$$

and, consequently, function $Q_{k}(t) u_{1}$ satisfies Eq. (4).

Let us check that if the function satisfies initial conditions (5) when $u_{0}=0$. Since when $t$ is small, there is the inequality

$$
\|S(t)\| \leq M \sinh t
$$

then, with respect to (13), when $t \rightarrow 0$ we get

$$
\begin{gathered}
\left\|Q_{k}(t)\right\| \leq M \nu_{k} \sinh ^{1-k} t \int_{0}^{t}(\cosh t-\cosh \tau)^{k / 2-1} \sinh \tau d \tau=M \nu_{k} \sinh ^{1-k} t \int_{1}^{\cosh t}(\cosh t-s)^{k / 2-1} d s \\
=M 2^{k} \sinh ^{1-k} t(\cosh t-1)^{k / 2}=M 2^{3 k / 2} \sinh ^{1-k} t \sinh ^{k}(t / 2) \leq M_{1} t \rightarrow 0,
\end{gathered}
$$

That is why function $Q_{k}(t) u_{1}$ satisfies the first condition from (5).

To check whether the function $Q_{k}(t) u_{1}$ satisfies the second condition from (5), we will derive formula (14) for its derivative. In order to do this we will rewrite (4) as

$$
\frac{1}{\sinh ^{k} t}\left(\sinh ^{k} t u^{\prime}(t)\right)^{\prime}+\frac{k^{2}}{4} u(t)=A u(t)+\frac{k \cosh ^{2-k}(t / 2)}{\sinh t} u^{\prime}(0) .
$$

Now let us substitute function $Q_{k}(t) u_{1}$ in this equation and integrate it after multiplying by $\sinh ^{k} t$ :

$$
\begin{array}{r}
\sinh ^{k} t Q_{k}^{\prime}(t) u_{1}=\int_{0}^{t} \sinh ^{k} s\left(A-\frac{k^{2}}{4} I\right) Q_{k}(t) u_{1} d s+k \int_{0}^{t} \sinh ^{k-1} s \cosh ^{2-k}(s / 2) d s u_{1} \\
=\int_{0}^{t} \sinh ^{k} s\left(A-\frac{k^{2}}{4} I\right) Q_{k}(t) u_{1} d s+k 2^{k-1} \int_{0}^{t} \sinh ^{k-1}(s / 2) \cosh (s / 2) d s u_{1}
\end{array}
$$




$$
=\int_{0}^{t} \sinh ^{k} s\left(A-\frac{k^{2}}{4} I\right) Q_{k}(t) u_{1} d s+2^{k} \sinh ^{k}(t / 2) u_{1} \text {. }
$$

Taking into account (13) and changing the order of integration, we will obtain

$$
\begin{gathered}
\sinh ^{k} t Q_{k}^{\prime}(t) u_{1}=\nu_{k} \int_{0}^{t} \sinh \tau \int_{0}^{\tau}(\cosh \tau-\cosh y)^{k / 2-1} S(y)\left(A-\frac{k^{2}}{4} I\right) u_{1} d y d \tau+2^{k} \sinh ^{k}(t / 2) u_{1} \\
=\nu_{k} \int_{0}^{t} S(y)\left(A-\frac{k^{2}}{4} I\right) u_{1} \int_{y}^{t} \sinh \tau(\cosh \tau-\cosh y)^{k / 2-1} d \tau d y+2^{k} \sinh ^{k}(t / 2) u_{1} \\
=\frac{2 \nu_{k}}{k} \int_{0}^{t}(\cosh \tau-\cosh y)^{k / 2} S(y)\left(A-\frac{k^{2}}{4} I\right) u_{1} d y+2^{k} \sinh ^{k}(t / 2) u_{1} \\
=\frac{2 \nu_{k}}{k \nu_{k+2}} \sinh ^{k+1} t Q_{k+2}(t)\left(A-\frac{k^{2}}{4} I\right) u_{1}+2^{k} \sinh ^{k}(t / 2) u_{1} .
\end{gathered}
$$

That is why the derivative of function $Q_{k}(t) u_{1}$ has the form (14), and function $Q_{k}(t) u_{1}$ satisfies the second condition from (5).

Theorem 3. Let $u_{0}, u_{1} \in D(A)$ and let operator $A$ be a generator of $C O F C(t)$. Then function $u(t)=P_{k}(t) u_{0}+Q_{k}(t) u_{1}$ is the unique solution to the Cauchy problem (4), (5).

Proof. The fact that function $u(t)=P_{k}(t) u_{0}+Q_{k}(t) u_{1}$ is a solution to problem (4), (5) is established in Theorems 1 and 2. We will prove the uniqueness of solution to problem (4), (5) by contradiction. Let $u_{1}(t)$ and $u_{2}(t)$ be the two solutions to problem (4), (5). Then function $v(t)=u_{1}(t)-u_{2}(t)$ satisfies Eq. (4) and conditions (5). By means of Theorem $1 v(t) \equiv 0$, and this proves the uniqueness of the solution and the theorem.

As it is established in [12], when $u_{0} \in E$ uniformly with respect to $t \in\left[0, t_{0}\right], t_{0}>0$,

$$
\lim _{k \rightarrow 0} P_{k}(t) u_{0}=C(t) u_{0}
$$

Analogously, for $u_{1} \in E$ we can obtain equation $\lim _{k \rightarrow 0} Q_{k}(t) u_{1}=S(t) u_{1}$. Consequently, there occurs the "coupling" of the resulting solution in Theorem 3 and the well-known solution to the Cauchy problem for abstract wave equation

$$
\lim _{k \rightarrow 0}\left(P_{k}(t) u_{0}+Q_{k}(t) u_{1}\right)=C(t) u_{0}+S(t) u_{1} .
$$

2. The boundary control problem for weakly loaded Legendre equation. We will seek solution $u(t) \in C^{2}([0,1], E) \cap C((0,1], D(A))$ to Eq. (4), satisfying two final conditions, given for the sake of convenience in point $t=1$

$$
u(1)=u_{2}, u^{\prime}(1)=u_{3} .
$$

As it follows from Theorem 3 , it suffices to define unknown initial elements $u_{0}, u_{1}$ in conditions (5) over final conditions (15) to justify the solvability of problem (4), (15). Applying conditions (15) to function $u(t)=P_{k}(t) u_{0}+Q_{k}(t) u_{1}$ and using Eqs. (6), (7), (13), (14) to find elements $u_{0}$, $u_{1}$ we will get the system

$$
\begin{gathered}
P_{k}(1) u_{0}+Q_{k}(1) u_{1}=u_{2} \\
\frac{\sinh 1}{k+1} P_{k+2}(1)\left(A-\frac{k^{2}}{4} I\right) u_{0}+\frac{\sinh 1}{k+2} Q_{k+2}(1)\left(A-\frac{k^{2}}{4} I\right) u_{1}+\frac{1}{\cosh ^{k} 1 / 2} u_{1}=u_{3} .
\end{gathered}
$$

It is convenient to rewrite Eqs. (16), (17) as a matrix equation

$$
B v=w, B: D(A) \times D(A) \longrightarrow E \times E,
$$


where

$$
\begin{aligned}
& v=\left(\begin{array}{l}
u_{0} \\
u_{1}
\end{array}\right), w=\left(\begin{array}{l}
u_{2} \\
u_{3}
\end{array}\right), \\
& B=\left(\begin{array}{cc}
B_{1} & B_{2} \\
B_{3} & B_{4}
\end{array}\right)=\left(\begin{array}{cc}
P_{k}(1) & Q_{k}(1) \\
\frac{\sinh 1}{k+1} P_{k+2}(1)\left(A-\frac{k^{2}}{4} I\right) & \frac{\sinh 1}{k+2} Q_{k+2}(1)\left(A-\frac{k^{2}}{4} I\right)+\frac{1}{\cosh ^{k} 1 / 2} I
\end{array}\right),
\end{aligned}
$$

wherein all operators $B_{1}, B_{2}, B_{3}, B_{4}$ commute on $D(A)$.

Thus the unique solvability of problem $(4),(15)$ reduces to the problem of existence of inverse operator matrix of operator matrix $B: D(A) \times D(A) \rightarrow E \times E$, given by (19), defined on some subset from $E \times E$. As in scalar case, an important role is played by the determinant of operator matrix $B$, which we will denote by $\Delta=B_{1} B_{4}-B_{2} B_{3}$.

Let $x \in D(A)$, with respect to (6) and (13), after elementary transformations we will get

$$
\begin{gathered}
\Delta x=P_{k}(1)\left(\frac{\sinh 1}{k+2} Q_{k+2}(1)\left(A-\frac{k^{2}}{4} I\right) x+\frac{1}{\cosh ^{k} 1 / 2} x\right)-\frac{\sinh 1}{k+1} Q_{k}(1) P_{k+2}(1)\left(A-\frac{k^{2}}{4} I\right) x \\
=\frac{1}{\sinh ^{k} 1} P_{k}(1) \int_{0}^{1} \sinh ^{k} s\left(A-\frac{k^{2}}{4} I\right) Q_{k}(s) x d s+\frac{1}{\cosh ^{k} 1 / 2} P_{k}(1) x \\
\quad-\frac{1}{\sinh ^{k} 1} Q_{k}(1) \int_{0}^{1} \sinh ^{k} s\left(A-\frac{k^{2}}{4} I\right) P_{k}(s) x d s \\
=\frac{1}{\sinh ^{k} 1} P_{k}(1) \int_{0}^{1} \sinh ^{k} s\left(Q_{k}^{\prime \prime}(s)+k \operatorname{coth} s Q_{k}^{\prime}(s)-k \frac{\cosh ^{2-k}(s / 2)}{\sinh s}\right) x+\frac{1}{\cosh ^{k} 1 / 2} P_{k}(1) x \\
\quad-\frac{1}{\sinh ^{k} 1} Q_{k}(1) \int_{0}^{1} \sinh ^{k} s\left(P_{k}^{\prime \prime}(s)+k \operatorname{coth} s P_{k}^{\prime}(s)\right) x \\
=P_{k}(1) Q_{k}^{\prime}(1) x-\frac{k}{\sinh ^{k} 1} P_{k}(1) x \int_{0}^{1} \sinh ^{k-1} s \cosh ^{2-k}(s / 2) d s+\frac{1}{\cosh ^{k} 1 / 2} P_{k}(1) x-Q_{k}(1) P_{k}^{\prime}(1) x \\
=P_{k}(1) Q_{k}^{\prime}(1) x-Q_{k}(1) P_{k}^{\prime}(1) x . \quad(20)
\end{gathered}
$$

Let us introduce

$$
W_{k}(t) x=\left|\begin{array}{cc}
P_{k}(t) & Q_{k}(t) \\
P_{k}^{\prime}(t) & Q_{k}^{\prime}(t)
\end{array}\right| x=P_{k}(t) Q_{k}^{\prime}(t) x-P_{k}^{\prime}(t) Q_{k}(t) x,
$$

the Wronski operator determinant built over operator functions $P_{k}(t)$ and $Q_{k}(t)$.

Thus, with respect to (20), the question of existence of inverse operator to $\Delta=B_{1} B_{4}-B_{2} B_{3}$ reduces to existence of operator which is inverse to Wronski operator determinant $W_{k}(1)$.

Lemma. Let $k>0, x \in D(A)$ and let operator $A$ be a generator of $C O F C(t)$. Then the Wronski operator determinant built over defined correspondingly by Eqs. (6), (13) operator functions $P_{k}(t)$ and $Q_{k}(t)$, equals

$$
W_{k}(t) x=\frac{k}{\sinh ^{k} t} \int_{0}^{t} \frac{\sinh ^{k-1} \tau}{\cosh ^{k-2} \tau / 2} P_{k}(\tau) x d \tau
$$

Proof. Let us show that function $W_{k}(t) x$ satisfies the equation

$$
W_{k}^{\prime}(t) x+k \operatorname{coth} t W_{k}(t) x=\frac{k \cosh ^{2-k} t / 2}{\sinh t} P_{k}(t) x
$$


and initial condition

$$
\lim _{t \rightarrow 0} W_{k}(t) x=x
$$

Indeed,

$$
\begin{gathered}
W_{k}^{\prime}(t) x=P_{k}^{\prime}(t) Q_{k}^{\prime}(t) x+P_{k}(t) Q_{k}^{\prime \prime}(t) x-P_{k}^{\prime}(t) Q_{k}^{\prime}(t) x-P_{k}^{\prime \prime}(t) Q_{k}(t) x \\
=P_{k}(t)\left(Q_{k}^{\prime \prime}(t)+k \operatorname{coth} t Q_{k}^{\prime}(t)-\frac{k \cosh ^{2-k} t / 2}{\sinh t} I+\frac{k^{2}}{4} Q_{k}(t)\right) x \\
-P_{k}(t)\left(k \operatorname{coth} t Q_{k}^{\prime}(t)-\frac{k \cosh ^{2-k} t / 2}{\sinh t} I+\frac{k^{2}}{4} Q_{k}(t)\right) x \\
-Q_{k}(t)\left(P_{k}^{\prime \prime}(t)+k \operatorname{coth} t P_{k}^{\prime}(t)+\frac{k^{2}}{4} P_{k}(t)\right) x+Q_{k}(t)\left(k \operatorname{coth} t P_{k}^{\prime}(t)+\frac{k^{2}}{4} P_{k}(t)\right) x \\
=P_{k}(t) A Q_{k}(t) x-Q_{k}(t) A P_{k}(t) x-k \operatorname{coth} t W_{k}(t) x+\frac{k \cosh ^{2-k} t / 2}{\sinh ^{2} P_{k}(t) x=} \\
=-k \operatorname{coth} t W_{k}(t) x+\frac{k \cosh ^{2-k} t / 2}{\sinh } P_{k}(t) x,
\end{gathered}
$$

and therefore the function $W_{k}(t) x$ satisfies Eq. (22).

When $P_{k}(0) x=Q_{k}^{\prime}(0) x=x, P_{k}^{\prime}(0) x=Q_{k}(0) x=0$, function $W_{k}(t) x$ also satisfies initial condition (23), and the unique solution to problem (22), (23) is a function defined by equality (21).

According to the lemma, we need to study the invertibility of bounded operator

$$
W_{k}(1) x=\frac{k}{\sinh ^{k} 1} \int_{0}^{1} \frac{\sinh ^{k-1} \tau}{\cosh ^{k-2} \tau / 2} P_{k}(\tau) x d \tau .
$$

Note that if $k=0$, then $W_{0}(t) x=C(t) S^{\prime}(t) x-C^{\prime}(t) S(t) x=C^{2}(t) x-A S^{2}(t) x=x$ and operator $W_{0}(t)=I$ is always invertible, but in general case $k>0$ it is not true, and the question of invertibility of operator $W_{k}(t)$ is very difficult. Hereinafter entire function

$\cosh i_{k}(\lambda)=\frac{k \mu_{k}}{\sinh ^{k} 1} \sum_{j=0}^{\infty} \frac{a_{j}(k)}{(2 j) !} \lambda^{j}=\frac{k \mu_{k}}{\sinh ^{k} 1} \int_{0}^{1} \cosh s \sqrt{\lambda} \int_{s}^{1} \cosh ^{2-k} \tau / 2(\cosh \tau-\cosh s)^{k / 2-1} d \tau d s$,

where $a_{j}(k)=\int_{0}^{1} s^{2 j} \int_{s}^{1} \cosh ^{2-k} \tau / 2(\cosh \tau-\cosh s)^{k / 2-1} d \tau d s$ will play an important role.

Theorem 4. Let $A$ be a bounded operator. For operator $W_{k}(1)$ defined by Eq. (24) to be invertible it is necessary and sufficient that on spectrum $\sigma(A)$ of operator A condition

$$
\cosh i_{k}(\lambda) \neq 0, \lambda \in \sigma(A)
$$

be satisfied.

Proof. We substitute (6) in (24), after elementary transformations we get

$$
W_{k}(1)=\frac{k \mu_{k}}{\sinh ^{k} 1} \sum_{j=0}^{\infty} \frac{1}{(2 j) !} A^{j} \int_{0}^{1} \cosh ^{2-k} \tau / 2 \int_{0}^{\tau} s^{2 j}(\cosh \tau-\cosh s)^{k / 2-1} d s d \tau=\cosh i_{k}(A) .
$$

Let $\Omega$ be an open set on complex plane containing spectrum $\sigma(A)$ of bounded operator $A$, boundary of which $\partial \Omega$ consists of finite number of rectifiable Jordan curves oriented in positive direction. Then by representing the operator from right-hand side of (27) through resolvent $R(\lambda)$ of operator $A$, we will get

$$
W_{k}(1)=\frac{1}{2 \pi i} \int_{\partial \Omega} \cosh i_{k}(\lambda) R(\lambda) d \lambda
$$


Necessary and sufficient condition of invertibility of operator $W_{k}(1)$ is absence of point $\lambda=0$ in spectrum $\sigma\left(W_{k}(1)\right)$ of operator $W_{k}(1)$. Eq. (28) means that operator $W_{k}(1)$ is an analytic function of operator $A, W_{k}(1)=\cosh i_{k}(A)$. By the theorem on the mapping of the spectrum of a bounded operator $\sigma\left(W_{k}(1)\right)=\cosh i_{k}(\sigma(A))$. Thus value $\lambda=0$ is not a point of the spectrum of operator $W_{k}(1)$ only when function $\cosh i_{k}(\lambda)$ does not vanishes on spectrum $\sigma(A)$ or, what is the same, condition $(26)$ is satisfied

$$
W_{k}^{-1}(1)=\frac{1}{2 \pi i} \int_{\partial \Omega} \frac{1}{\cosh i_{k}(\lambda)} R(\lambda) d \lambda .
$$

From Theorem 4 it follows that the location of zeros of function $\cosh i_{k}(\lambda)$ defines the invertibility of operator $W_{k}(1)$ in the case of bounded operator $A$. In the case of unbounded operator $A$ condition of the form (26) will not be sufficient condition of invertibility, despite the zeros location will still play an important role.

Let us consider the case when in Eq. (4) parameter $k=2$. In this case

$$
\cosh i_{2}(\lambda)=\frac{2}{\sinh ^{2} 1} \frac{\cosh \lambda-1}{\lambda^{2}},
$$

zeros of function $\cosh i_{2}(\lambda)$ can be calculated using the formula

$$
\lambda_{j}=j \frac{\pi i}{2}, j \in \mathbb{Z} \backslash\{0\},
$$

let us indicate the sufficient condition of invertibility of operator $W_{k}(1)$ in the case of unbounded operator $A$.

We denote by $\Upsilon_{0}$ the contour on complex plane consisting of straight line $\operatorname{Re} z=\sigma_{0}>\omega$ (we pass it bottom-up), $\omega$ is a $C(t)$ COF growth exponent, $\Upsilon_{0}^{2}$ is a parabola, image $\Upsilon_{0}$ under mapping $w=z^{2}$ $\left(z \in \Upsilon_{0}, w \in \Upsilon_{0}^{2}\right)$.

There can be only finite number of zeros $\lambda_{j}$ to the left of parabola $\Upsilon_{0}^{2}$, we will denote their set by $\Lambda$, $\operatorname{card}(\Lambda)<\infty$.

Condition 1. Let $k=2$ and each zero $\lambda_{j}$ of entire function $\cosh i_{2}(\lambda)$, defined by Eq. (31), which lies to the left of parabola $\Upsilon_{0}^{2}$, belong to resolvent set $\rho(A)$ of operator $A$ and there exists $d>0$ such that $\max _{j \in \Lambda}\left\|R\left(\lambda_{j}\right)\right\| \leq d$.

We assume that Condition 1 is satisfied. Since each zero $\lambda_{j} \in \Lambda$ belongs to $\rho(A)$, it belongs to $\rho(A)$ with circular neighborhood $\Omega_{j}$ of radius $1 / d$, boundary of which (we pass it clockwise) we denote by $\gamma_{j}$ and let

$$
\Xi=\Upsilon_{0}^{2} \bigcup \bigcup_{j \in \Lambda} \gamma_{j}
$$

Our problem reduces to a problem of existence of an operator defined on some subset of $D(A)$ which is inverse operator to the bounded operator given by Eq. (28) when $k=2$ and continuously extended on $E$. In order to do it when $x \in E, \lambda_{0} \in \mathbb{C}$ we introduce bounded operator

$$
H x=\frac{1}{2 \pi i} \int_{\Xi} \frac{R(z) x d z}{\cosh i_{2}(z)\left(z-\lambda_{0}\right)^{3}}, \quad H: E \rightarrow E .
$$

Let us show that the integral in (32) converges absolutely when Condition 1 is satisfied. Indeed, by virtue of the choice of contour $\Upsilon_{0}^{2}$, inequality [13]

$$
\left\|\lambda R\left(\lambda^{2}\right)\right\| \leq \frac{M}{\operatorname{Re} \lambda-\omega}, \quad \operatorname{Re} \lambda>\omega,
$$

and the boundedness of function $\left(\cosh \lambda^{2}-1\right)^{-1}$ integral

$$
\int_{\Upsilon_{0}^{2}} \frac{R(z) d z}{\cosh i_{2}(z)\left(z-\lambda_{0}\right)^{3}}=2 \int_{\Upsilon_{0}} \frac{\lambda R\left(\lambda^{2}\right) d \lambda}{\cosh i_{2}\left(\lambda^{2}\right)\left(\lambda^{2}-\lambda_{0}\right)^{3}}=2 \int_{\Upsilon_{0}} \frac{\lambda^{5} R\left(\lambda^{2}\right) d \lambda}{\left(\cosh \lambda^{2}-1\right)\left(\lambda^{2}-\lambda_{0}\right)^{3}}
$$


converges absolutely.

Theorem 5. Let operator $A$ be a generator of COF $C(t), x \in D\left(A^{4}\right)$ and Condition 1 is satisfied. Then operator $W_{k}(1)$ has inverse operator $W_{k}^{-1}(1): D\left(A^{3}\right) \rightarrow E$.

Proof. Let $x \in D(A), \sigma_{0}<\sigma<\operatorname{Re} \xi$. Then by substituting operator $W_{k}(1)$ defined by Eq. (28) in (32) and applying the Hilbert identity

$$
R(z) R\left(\xi^{2}\right)=\frac{R(z)-R\left(\xi^{2}\right)}{\xi^{2}-z}
$$

we get

$$
\begin{aligned}
& H W_{2}(1) x=\frac{1}{2 \pi i} \int_{\Xi} \frac{R(z)}{\cosh i_{2}(z)\left(z-\lambda_{0}\right)^{3}} \frac{1}{i \pi} \int_{\sigma-i \infty}^{\sigma+i \infty} \cosh i_{2}\left(\xi^{2}\right) \xi R\left(\xi^{2}\right) x d \xi d z \\
& \quad=-\frac{1}{2 \pi^{2}} \int_{\Xi} \int_{\sigma-i \infty}^{\sigma+i \infty}\left(\frac{\xi \cosh i_{2}\left(\xi^{2}\right) R(z) x}{\cosh i_{2}(z)\left(z-\lambda_{0}\right)^{3}\left(\xi^{2}-z\right)}-\frac{\xi \cosh i_{2}\left(\xi^{2}\right) R\left(\xi^{2}\right) x}{\cosh i_{2}(z)\left(z-\lambda_{0}\right)^{3}\left(\xi^{2}-z\right)}\right) d \xi d z .
\end{aligned}
$$

The integral in (33) converges absolutely. By changing the order of integration we will obtain

$$
\begin{aligned}
H W_{2}(1) x=-\frac{1}{2 \pi^{2}} \int_{\Xi} & \int_{\sigma-i \infty}^{\sigma+i \infty} \frac{\xi \cosh i_{2}\left(\xi^{2}\right) R(z) x d \xi d z}{\cosh i_{2}(z)\left(z-\lambda_{0}\right)^{3}\left(\xi^{2}-z\right)} \\
& +\frac{1}{2 \pi^{2}} \int_{\sigma-i \infty}^{\sigma+i \infty} \xi \cosh i_{2}\left(\xi^{2}\right) R\left(\xi^{2}\right) x \int_{\Xi} \frac{d z}{\cosh i_{2}(z)\left(z-\lambda_{0}\right)^{3}\left(\xi^{2}-z\right)} d \xi .
\end{aligned}
$$

If we close integration contour $\Upsilon_{0}^{2}$ to the left without intersection with $\cup_{j \in \Lambda} \gamma_{j}$, then the inner integral in the second summand in (34) vanishes by virtue of choice of contour $\Xi$ and the Cauchy theorem for multiply connected domains. For calculations of the integrals in the first summand in (34) we will use the Cauchy integral formula. Thus we get

$$
\begin{aligned}
& H W_{2}(1) x=-\frac{1}{2 \pi^{2}} \int_{\Xi} \int_{\Upsilon} \frac{\xi \cosh i_{2}\left(\xi^{2}\right) R(z) x d \xi d z}{\cosh i_{2}(z)\left(z-\lambda_{0}\right)^{3}\left(\xi^{2}-z\right)} \\
& =-\frac{1}{4 \pi^{2}} \int_{\Xi} \int_{\Upsilon^{2}} \frac{\cosh i_{2}(\lambda) R(z) x d \lambda d z}{\cosh i_{2}(z)\left(z-\lambda_{0}\right)^{3}(\lambda-z)}=\frac{1}{2 \pi i} \int_{\Xi} \frac{R(z) x d z}{\left(z-\lambda_{0}\right)^{3}}=\frac{1}{2 \pi i} \int_{\Upsilon_{0}^{2}} \frac{R(z) x d z}{\left(z-\lambda_{0}\right)^{3}} \\
& =-\frac{1}{2} R^{\prime \prime}\left(\lambda_{0}\right) x=-R^{3}\left(\lambda_{0}\right) x .
\end{aligned}
$$

Commuting operators $H, W_{2}(1), R^{3}\left(\lambda_{0}\right)$ are bounded and domain $D(A)$ is dense in $E$, that is why equation $H W_{2}(1) x=-R^{3}\left(\lambda_{0}\right) x$ is fair for $x \in E$, herewith $H W_{2}(1): E \rightarrow D\left(A^{3}\right)$. Consequently, the operator

$$
W_{2}^{-1}(1) x=-\left(\lambda_{0} I-A\right)^{3} H x, W_{2}^{-1}(1): D\left(A^{3}\right) \rightarrow E,
$$

is an inverse operator for $W_{k}(1)$. Indeed,

$$
\begin{gathered}
W_{2}(1) W_{2}^{-1}(1) x=-W_{2}(1)\left(\lambda_{0} I-A\right)^{3} H x=-W_{2}(1) H\left(\lambda_{0} I-A\right)^{3} x \\
=R^{3}\left(\lambda_{0}\right)\left(\lambda_{0} I-A\right)^{3} x=x, \quad x \in D\left(A^{3}\right), \\
W_{2}^{-1}(1) W_{2}(1) x=-\left(\lambda_{0} I-A\right)^{3} H W_{2}(1) x=\left(\lambda_{0} I-A\right)^{3} R^{3}\left(\lambda_{0}\right) x=x, x \in E .
\end{gathered}
$$

In Theorems 4 and 5 we use the set on which operator $W_{k}(1)$ has inverse operator $W_{k}^{-1}(1)$ which has the form (29) in the case of bounded operator $A$ and $k>0$ and the form (35) in the case of unbounded 
operator $A$ and $k=2$. Thus by virtue of (20) we proved the existence of the inverse operator to $\Delta$. By solving matrix Eq. (18), like in the scalar case we will get

$$
\left(\begin{array}{l}
u_{0} \\
u_{1}
\end{array}\right)=\Delta^{-1}\left(\begin{array}{cc}
B_{4} & -B_{3} \\
-B_{2} & B_{1}
\end{array}\right)\left(\begin{array}{l}
u_{2} \\
u_{3}
\end{array}\right) .
$$

Thereby, by virtue of Theorems 4 and 5 we can obtain the following statements on solvability of the boundary control problem considered in this Item in which function $\cosh i_{k}(\lambda)$ from (25) plays a great role.

Theorem 6. Let $u_{2}, u_{3} \in E, A$ be a bounded operator and condition $\cosh i_{k}(\lambda) \neq 0, \lambda \in \sigma(A)$ be satisfied on spectrum $\sigma(A)$ of operator $A$. Then problem (4), (15) has unique solution $u(t)=$ $P_{k}(t) u_{0}+Q_{k}(t) u_{1}$, where

$$
\begin{gathered}
u_{0}=W_{k}^{-1}(1)\left(\frac{\sinh 1}{k+2} Q_{k+2}(1)\left(A-\frac{k^{2}}{4} I\right) u_{2}+\frac{u_{2}}{\cosh ^{k} 1 / 2}-\frac{\sinh 1}{k+1} P_{k+2}(1)\left(A-\frac{k^{2}}{4} I\right) u_{3}\right), \\
u_{1}=W_{k}^{-1}(1)\left(-Q_{k}(1) u_{2}+P_{k}(1) u_{3}\right),
\end{gathered}
$$

and operator $W_{k}^{-1}(1)$ has the form (29).

Theorem 7. Let $u_{2}, u_{3} \in D\left(A^{4}\right)$ and Condition 1 be satisfied. Then problem (4), (15) has unique solution $u(t)=P_{2}(t) u_{0}+Q_{2}(t) u_{1}$, where initial elements $u_{0}, u_{1}$ are defined in (36), (37) when $k=2$, and

$$
W_{2}^{-1}(1) x=-\frac{1}{2 \pi i} \int_{\Xi} \frac{R(z)\left(\lambda_{0} I-A\right)^{3} x d z}{\cosh i_{2}(z)\left(z-\lambda_{0}\right)^{3}}, x \in D\left(A^{3}\right) .
$$

3. Nonlocal problem for the Legendre equation. We will seek solution $u(t) \in C^{2}([0,1], E) \cap$ $C((0,1], D(A))$ to Eq. (1) that satisfies the nonlocal integral condition with fractional integral $I_{g}^{\beta}, \beta>0$ with respect to function $g(t)=\cosh t$,

$$
\lim _{t \rightarrow 1} I_{g}^{\beta}\left(\sinh ^{k-1} t u(t)\right)=u_{4},
$$

and the condition

$$
u^{\prime}(0)=0 .
$$

Problem (1), (38), (39) with nonlocal conditions (38), (39), broadly speaking, is not correct. Let us specify the conditions on operator $A$ and element $u_{4} \in E$ providing its unique solvability.

Among publications on solvability of nonlocal problems with integral condition for abstract differential first-order equations we will note papers [20] and [21]. The criterion of the uniqueness of solution is established in [22]. The nonlocal problem for Euler-Poisson-Darboux equation is studied in [23].

The research on solvability of nonlocal problem (1), (38), (39) are based on locating of initial element $u_{0}$ in condition (2) using nonlocal condition (38).

We apply the fractional integral with respect to function $g(t)=\cosh t$ to function $u(t)=P_{k}(t) u_{0}$, multiplied by $\sinh ^{k-1} t$, where $P_{k}(t)$ is defined by Eq. (6). Taking into account the semigroup property of fractional integration and condition (38), we get

$$
\begin{aligned}
\lim _{t \rightarrow 1} I_{g}^{\beta}\left(\sinh ^{k-1} t u(t)\right)=\mu_{k} \Gamma(k / 2) \lim _{t \rightarrow 1} I_{g}^{k / 2+\beta}\left[\frac{C(t)}{\sinh t}\right] u_{0} \\
=\frac{\mu_{k} \Gamma(k / 2)}{\Gamma(k / 2+\beta)} \int_{0}^{1}(\cosh 1-\cosh s)^{k / 2+\beta-1} C(s) u_{0} d s=u_{4} .
\end{aligned}
$$


As before, when establishing the solvability of nonlocal problem (1), (38), (39) we will use entire function

$$
\psi_{k, \beta}(\lambda)=\frac{\mu_{k} \Gamma(k / 2)}{\Gamma(k / 2+\beta)} \sum_{j=0}^{\infty} \frac{b_{j}(k, \beta)}{(2 j) !} \lambda^{j}=\frac{\mu_{k} \Gamma(k / 2)}{\Gamma(k / 2+\beta)} \int_{0}^{1} \cosh s \sqrt{\lambda}(\cosh 1-\cosh s)^{k / 2+\beta-1} d s,
$$

where

$$
b_{j}(k, \beta)=\int_{0}^{1} s^{2 j}(\cosh 1-\cosh s)^{k / 2+\beta-1} d s .
$$

Theorem 8. Let $A$ be a bounded operator and $u_{4} \in$ E. In order for problem (1), (38), (39) to have unique solution it is necessary and sufficient that condition $\psi_{k, \beta}(\lambda) \neq 0, \lambda \in \sigma(A)$ be satisfied on spectrum $\sigma(A)$ of operator A. Herewith $u(t)=P_{k}(t) u_{0}$, where

$$
u_{0}=\frac{1}{2 \pi i} \int_{\partial \Omega} \frac{1}{\psi_{k, \beta}(\lambda)} R(\lambda) u_{4} d \lambda
$$

Proof is analogous to the proof of theorem 4.

Let us consider the case in which $\beta=1-k / 2,0<k \leq 2$ :

$$
\psi_{k}(\lambda)=\frac{\mu_{k} \Gamma(k / 2) \sinh \sqrt{\lambda}}{\sqrt{\lambda}},
$$

zeros $\lambda_{j}$ of function $\psi_{k}(\lambda)$ can be calculated explicitly:

$$
\lambda_{j}=-\pi^{2} j^{2}, \quad j \in \mathbb{N} .
$$

Let us specify the sufficient solvability condition of nonlocal problem (1), (38), (39) in the case of unbounded operator $A$.

Condition 2. Let each zero $\lambda_{j}=-\pi^{2} j^{2}, j \in \mathbb{N}$ of a function $\psi_{k}(\lambda)$ belong to resolvent set $\rho(A)$ and there exists $d>0$ such that $\sup _{j=1,2}\left\|R\left(\lambda_{j}\right)\right\| \leq d$.

Since each zero $\lambda_{j}, j=1,2, \ldots$ of function $\cosh i_{k}(\lambda)$ belongs to $\rho(A)$, it belongs to $\rho(A)$ with a circular neighborhood $\Omega_{j}$ of radius $\frac{1}{d}$, boundary of which (we pass it clockwise) we denote by $\gamma_{j}$. Let $\Upsilon_{0}$ be a contour on complex plane consisting of straight line (we pass it bottom-up) $\operatorname{Re} z=\sigma_{0}>\omega, \Upsilon_{0}^{2}$ be a parabola, image $\Upsilon_{0}$ under mapping $w=z^{2}\left(z \in \Upsilon_{0}, w \in \Upsilon_{0}^{2}\right)$, and $\Xi=\Upsilon_{0}^{2} \underset{j=1,2, \ldots}{\cup} \gamma_{j}$.

We put $\lambda_{0} \in \rho(A), \operatorname{Re} \lambda_{0}>\sigma>\sigma_{0}$ and introduce bounded operator

$$
H v=\frac{1}{2 \pi i} \int_{\Xi} \frac{R(z) v d z}{\psi_{k}(z)\left(z-\lambda_{0}\right)^{2}}, H: E \rightarrow E .
$$

As in the proof of Theorem 5 we establish that the integral in (40) under Condition 2 converges absolutely and there is valid

Theorem 9. Let operator $A$ be a generator of $\operatorname{COF} C(t), x \in D\left(A^{3}\right)$ and Condition 2 be satisfied. Then problem (1), (38), (39) is uniquely solvable and the solution has he form $u(t)=P_{k}(t) u_{0}$, where $u_{0}=\left(\lambda_{0} I-A\right)^{2} H u_{4}$, and operator $H$ is defined in (40).

\section{ACKNOWLEDGMENTS}

This paper is financially supported by Russian Foundation for Basic Research, grant No. 16-0100197 A-2016. 


\section{REFERENCES}

1. Carroll, R. W., Showalter, R. E. Singular and Degenerate Cauchy Problems (Academic Press, N. Y., 1976).

2. Glushak, A. V., Pokruchin, O. A. "Criterion for the Solvability of the Cauchy Problem for an Abstract EulerPoisson-Darboux Equation”, Differ. Equ. 52, No. 1, 39-57 (2016).

3. Glushak, A. V. "The Bessel Operator Function", Dokl. Math. 55, No. 1, 103-105 (1997).

4. Bateman, H., Erdelyi, A. Higher Transcendental Functions (MC Graw-Hill company, inc., New York, 1967), Vol. 3.

5. Helgason, S. Groups and Geometric Analysis (Academic press, Orlando, 1984).

6. Olevskii, M. N. "On Connections Between Solutions of the Generalized Wave Equation and the Generalized Heat-Conduction Equation”, Dokl. Akad. Nauk SSSR 101 (1), 21-24 (1955).

7. Yaroslavtseva, V. Ya. "On One Transformation Operator Class and its Applications to Differential Equations", Dokl. Akad. Nauk SSSR 227 (4), 816-819 (1976) [Russian].

8. Kipriyanov, I. A., Ivanov, L. A. "The Euler-Poisson-Darboux Equation in a Riemannian Space”, Sov. Math., Dokl. 24, 331-335 (1981).

9. Kipriyanov, I. A., Ivanov, L. A. "The Cauchy problem for the Euler-Poisson-Darboux equation in a homogeneous symmetric Riemannian space. I”, Proc. Steklov Inst. Math. 170, 159-168 (1987).

10. Kipriyanov, I. A., Ivanov, L. A. "The Cauchy Problem for the Euler-Poisson-Darboux Equation in a Symmetric Space", Mathematics of the USSR-Sbornik 52, No. 1, 41-51 (1985).

11. Kononenko, V. I., Khinkis, L. A. "Transformation Operators Related to the Jacobi Differential Operator", No. 1604-B89 (Voronezh University, Voronezh, 1989) [Russian].

12. Glushak, A. V. “The Legendre Operator Function”, Izv. Math. 65, No. 6, 1073-1083 (2001).

13. Vasil'ev, V. V., Krein, S. G., Piskarev, S. I. "Operator Semigroups, Cosine Operator Functions, and Linear Differential Equations”, J. Sov. Math., Collection: Mathematical Analysis 54, 1042-1129 (1991).

14. Vasil'ev, V. V., Piskarev, S. I. "Differential Equations in Banach Spaces II. Cosine-Operator Functions", http://www.srcc.msu.su/nivc/english/about/home_pages/piskarev/obz2ru.pdf

15. Glushak, A. V. "Stabilization of Solution of the Cauchy Problem for an Abstract Differential Equation of the First Order", Russian Mathematics 45, No. 11, 1-11 (2001).

16. Dzenaliev, M. T., Ramazanov, M. I. Loaded Equations as the Perturbations of Differential Equations (Fylym, Almaty, 2010).

17. Nakhushev, A. M. Loaded Equations and Their Applications (Nauka, Moscow, 2012) [Russian]

18. Samko, S. G., Kilbas, A. A., Marichev, O. I. Fractional Integrals and Derivatives: Theory and Applications (CRC Press, Boca Raton, 1993).

19. Prudnikov, A. P., Brychkov, Yu. A., Marichev, O. I. Integrals and Series: Special Functions (CRC Press, Boca Raton, 1986).

20. Tikhonov, I. V. "Solvability of a Problem With a Nonlocal Integral Condition for a Differential Equation in a Banach Space,”, Differ. Equations 34, No. 6, 841-844 (1998).

21. Silchenko, Yu. T. "A Parabolic Equation With Nonlocal Conditions", Journal of Mathematical Sciences 149, No. 6, 1701-1707 (2008).

22. Tikhonov, I. V. "Uniqueness Theorems in Linear Nonlocal Problems for Abstract Differential Equations", Izv. Math. 67, No. 2, 333-363 (2003).

23. Glushak, A. V. "Abstract Euler-Poisson-Darboux Equation With Nonlocal Condition", Russian Mathematics 60, No 6, 21-28 (2016).

Translated by D. B. Katz 